\title{
La política de mitigación de los riesgos en las laderas de Quito: ¿qué vulnerabilidad combatir?
}

La politique de réduction des risques sur les versants de Quito : quelle vulnérabilité combattre?

Reduction risk policy on urban slopes of Quito: what vulnerability to fight against?

\section{Alexis Sierra}

\section{(2) OpenEdition}

Journals

\section{Edición electrónica}

URL: http://journals.openedition.org/bifea/2421

DOI: $10.4000 /$ bifea.2421

ISSN: 2076-5827

Editor

Institut Français d'Études Andines

\section{Edición impresa}

Fecha de publicación: 1 diciembre 2009

Paginación: 737-753

ISSN: 0303-7495

Referencia electrónica

Alexis Sierra, « La política de mitigación de los riesgos en las laderas de Quito: ¿qué vulnerabilidad combatir? », Bulletin de l'Institut français d'études andines [En línea], 38 (3) | 2009, Publicado el 01 junio 2010, consultado el 17 noviembre 2020. URL : http://journals.openedition.org/bifea/2421 ; DOI : https://doi.org/10.4000/bifea.2421

\section{@ $\odot \Theta \Theta$}

Les contenus du Bulletin de l'Institut français d'études andines sont mis à disposition selon les termes de la licence Creative Commons Attribution - Pas d'Utilisation Commerciale - Pas de Modification 4.0 International. 


\section{PARTE 3}

\section{REDUCCIÓN DE LA VULNERABILIDAD: POLÍTICAS URBANAS}

RÉDUCTION DE LA VULNÉRABILITÉ :

POLITIQUES URBAINES

\section{VULNERABILITY REDUCTION:}

\section{URBAN POLICIES}





\title{
La política de mitigación de los riesgos en las laderas de Quito: iqué vulnerabilidad combatir?
}

\author{
Alexis Sierra*
}

\section{Resumen}

El tratamiento y el acondicionamiento de las laderas occidentales de la ciudad constituyen el núcleo de la gestión del riesgo de origen natural en Quito. La deconstrucción de esta política revela tanto una representación de los desafíos de desarrollo como una lógica de control de las márgenes urbanas. En efecto, el acondicionamiento de las laderas ha llevado a luchar contra amenazas que ponen en peligro el funcionamiento de la red de saneamiento y la movilidad urbana. Desde antes, este se inscribe dentro de una lógica de control de la expansión urbana y de territorialización de las márgenes, pues las autoridades desean integrarlas a la planificación municipal. La evolución de la obra muestra también que la lucha contra el riesgo «natural» sirve a la política de mejoramiento del marco de vida.

Palabras clave: gestión del riesgo, margen urbana, Quito, vulnerabilidad

\section{La politique de réduction des risques sur les versants de Quito : quelle vulnérabilité combattre?}

\section{Résumé}

Le traitement et I'aménagement des versants occidentaux de la ville sont au cœur de la gestion du risque d'origine naturelle à Quito. La déconstruction de cette politique publique révèle autant une représentation des enjeux de développement qu'une logique de maîtrise des marges urbaines. En effet, l'aménagement des versants a conduit à lutter contre des aléas qui mettent en danger le fonctionnement du réseau d'assainissement et la mobilité urbaine. Plus en amont, il s'inscrit dans

* Université de Cergy-Pontoise-IUFM, Institut de Recherche pour le Développement (IRD), UR 029, programa Pacivur, 32 avenue Varagnat, 93143 Bondy Cedex, France.

E-mail: alexisierra2001@yahoo.fr 
une logique de contrôle de l'expansion urbaine et de territorialisation de marges que les autorités souhaitent intégrer à la planification municipale. L'évolution du chantier montre également que la lutte contre le risque «naturel » sert une politique d'amélioration du cadre de vie.

Mots clés : gestion du risque, marge urbaine, Quito, vulnérabilité

\title{
Reduction risk policy on urban slopes of Quito: what vulnerability to fight against?
}

\begin{abstract}
The treatment and planning of the western slopes of the Quito are at the heart of the natural risk management in the city. The deconstruction of this public policy reveals as much about the challenges of development as it does about the logic of controlling urban margins. It is true that the planning of the slopes has led to a fight against hazards that endanger the operation of the sanitation network and urban mobility. Even before, this was part of a logic of control of urban sprawl and territorialization of margins that the authorities wish to integrate into the municipal planning. The evolution of this project also shows that the struggle against the "natural" risk supports a policy to improvement the quality of life.
\end{abstract}

Key words: risk management, deprived urban area, Quito, vulnerability

\section{INTRODUCCIÓN}

Quito es una capital de 1,5 millones de habitantes afectada por eventos hidromorfoclimáticos recurrentes que han provocado, en un siglo, un poco menos de 600 accidentes con un total aproximado de 200 víctimas y daños materiales difícilmente evaluables (Peltre, 1989)1. El número de víctimas resulta escaso comparado con otros tipos de accidentes urbanos, pero los eventos hidromorfoclimáticos crean disfunciones recurrentes. Muy en particular, es el caso de las lavas torrenciales (Perrin et al., 2000). Estas perturban sobremanera la movilidad urbana y el funcionamiento de la red de evacuación de las aguas, considerados ambos como bienes comunes esenciales del medio ambiente urbano (Metzger \& Peltre, 1996). Estos dos elementos de desarrollo urbano determinan por antonomasia la representación del riesgo y de sus espacios (Sierra, 2000; 2003). El riesgo hídrico está estrechamente asociado a la pérdida de la movilidad. Ahora bien, la red combinada de evacuación de las aguas, pluvial y de saneamiento, está en el corazón de esta perturbación, ya que no permite drenar las aguas pluviales en caso de crecida repentina. Las aguas desbordan por las tapas de caja y los sumideros: las inundaciones de la calzada son recurrentes en esta ciudad andina. Así, la presencia de la red de evacuación de las aguas y su subdimensionamiento determinan la amenaza. En sentido contrario, esta red se daña por las crecidas de lodo que la obstruyen. En la entrada de la red combinada, las quebradas han sido

1 Censo efectuado en un periodo que va de 1900 a 1988 y que atañe a los accidentes ligados a los movimientos de masa y las dinámicas de flujos de lodo en la crecida torrencial. 
rellenadas. Los flujos generados arriba de las quebradas abiertas bajan entonces rápidamente por las calles paralelas a la pendiente, Ilegando a los barrios centrales y perturbando la movilidad. Los daños a estos dos bienes comunes representan una vulnerabilidad para la ciudad en su conjunto y han llevado a las autoridades públicas a movilizar importantes medios técnicos y financieros para luchar contra las amenazas hídricas y morfoclimáticas.

Es así como desde hace unos diez años, las autoridades municipales han aplicado un programa de acondicionamiento de las laderas occidentales de la ciudad, espacio de génesis de las crecidas. Esta obra comprende tres etapas, que corresponden a tres sectores de las laderas. Entre 1997 y 2002 se ha dado prioridad a las laderas noroccidentales. De 2002 a 2007 fueron tratadas las laderas situadas arriba del centro histórico. Una tercera etapa fue lanzada en 2008, a lo largo de las laderas sudoccidentales. Entre la primera etapa y las siguientes, esta política de tratamiento de los espacios de amenazas ha sufrido una inflexión, pasando a ser más global y menos ingenierista, aun cuando esta última dimensión sigue siendo central. La primera etapa fue analizada de manera precisa en una tesis de doctorado (Sierra, 2000) y las dos otras lo fueron dentro de las investigaciones efectuadas desde 2007 en el marco del programa Pacivur2.

El análisis de esta política de reducción de los riesgos muestra que son menos las amenazas, muy reales, que los desafíos de desarrollo y el tratamiento de las márgenes urbanas, los que explican las opciones de gestión y de acondicionamiento realizadas. Aunque el desarrollo de esta política sufre inflexiones en la última década, esta se halla inmersa en una lógica histórica de planificación y de relación de la ciudad con sus márgenes. Exponemos así las conclusiones de esta investigación, abordando en un primer momento el estudio de las diferentes etapas de la obra efectuada desde 1998, y en una segunda parte los desafíos políticos y territoriales que se desprenden de la misma.

\section{DE UNA RESPUESTA DE INGENIERÍA CIVIL A LA TENTATIVA DE ACONDICIONAMIENTO INTEGRAL DEL TERRITORIO}

\section{1. El acondicionamiento de las laderas noroccidentales desde 1998}

Entre las quebradas de Miraflores y de El Rancho, espacio que incluye a 32 quebradas, la municipalidad, a través de su empresa de aducción y de saneamiento de aguas (Emaap-Q3), ha realizado una inmensa obra de acondicionamiento de las quebradas. Esta ha comprendido 83 grandes obras de ingeniería, entre las cuales se encuentran dos presas de embalse de cemento armado de una capacidad de $157000 \mathrm{~m}^{3}$, diez presas de tierra, 54 diques y rejillas para la retención de

2 Programa andino de capacitación e investigación sobre vulnerabilidad y riesgos en medio urbano.

3 Empresa Municipal de Alcantarillado y Agua Potable de Quito. 
lodo, 2000 m de túneles de desvío de las aguas, la canalización parcial de algunas quebradas, la transformación de los fondos de talweg mediante graderías de ruptura de los flujos o de reservorios de protección a la entrada de la red de saneamiento urbano. El conjunto de la primera etapa representó cerca de 26 millones de dólares, cifra equivalente en el momento del voto en 1997 a 3/4 del presupuesto anual de la municipalidad. El financiamiento solo fue posible mediante el acuerdo de un préstamo negociado entre la Emaap-Q y el Banco Interamericano de Desarrollo. Más de la mitad de este presupuesto se destinó a la construcción de obras de protección, solamente un poco más del $6 \%$ al monitoreo, a la gestión de los residuos y a la capacitación de la población; el resto sirvió para los estudios y los gastos administrativos $y$ financieros.

La mayoría de las obras apuntaban inicialmente a la reducción del volumen de las aguas que descienden por las laderas. En un segundo momento, también fueron contempladas las presas de retención de las crecidas de lodo, siguiendo la idea de que el sistema de evacuación de las aguas pluviales era insuficiente para drenar el agua proveniente de las laderas. Estas obras traducen la representación inicial de la amenaza, es decir las inundaciones, generalmente considerada cuando perturba la movilidad urbana. Los estudios mostraron después que la infiltración sobre estas laderas era buena y que la causante no era tanto la cantidad de las escorrentías sino la existencia de lavas torrenciales4. Sin embargo, las obras diseñadas inicialmente fueron construidas y parecen a veces sobredimensionadas en relación con la realidad de los caudales (fig. 1).

El seguimiento de esta política de acondicionamiento parece haber tenido en cuenta esta visión parcial de la lucha contra el riesgo morfoclimático, al combinar respuesta de ingeniería civil y tratamiento social del espacio.

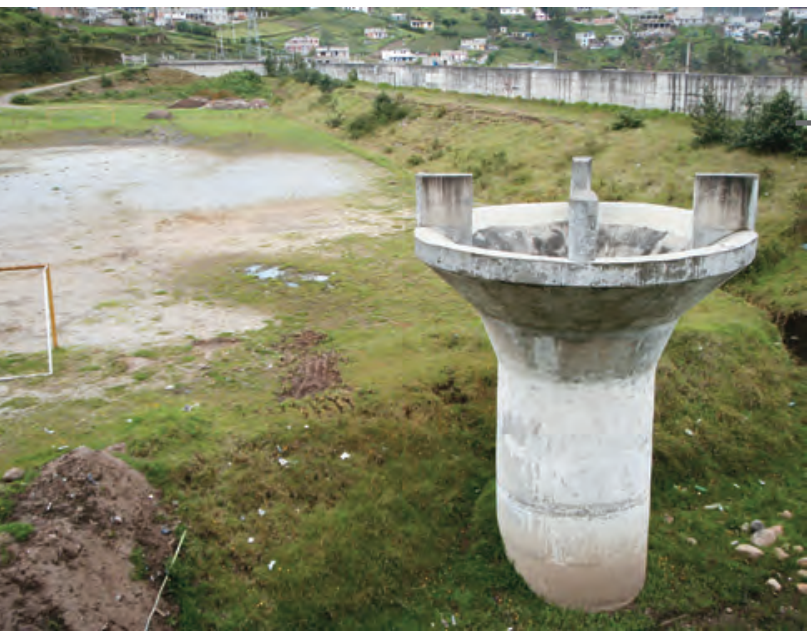

Figura 1 - Obras de protección en la quebrada Rumihurcu

El muro que aparece en el segundo plano sirve de retención en caso de crecida. En primer plano, el Tulipán de cemento es un desfogue que sirve para evacuar el exceso de agua en caso de que la crecida de las aguas alcance la cima de la presa. El conducto vertical conduce a un colector subterráneo. Según los estudios hidrológicos efectuados, ninguna crecida hídrica debería llegar a tal nivel y una lava torrencial provocaría rápidamente su obstrucción.

Foto: A. Sierra, marzo de 2008

4 Estudios efectuados de 1996 a 2000 por el programa de investigación Sishilad (IRD-Emaap). 


\section{2. El acondicionamiento de las laderas centrales (2002-2007)}

Luego de la primera fase, el programa «Laderas del Pichincha» fue integrado a un nuevo servicio de la Emaap-Q, el Programa de Saneamiento Ambiental (PSA), que agrupa la protección y el desarrollo de la red de saneamiento. En el tratamiento de las laderas centrales están claramente menos presentes las obras hidráulicas de lucha contra los fenómenos morfoclimáticos. Estas consisten en el reforzamiento de la red de saneamiento y en la modificación puntual de las quebradas (realización de cunetas, de pequeños reservorios y de algunos diques). El acondicionamiento ha consistido sobre todo en mejorar las condiciones de vida de la población, mediante la apertura de tres parques urbanos, de áreas de juego y de descanso, de miradores, de circuitos pedestres y a través de la construcción de puentes peatonales y de escalinatas que faciliten el acceso en los barrios (fig. 2).

Esta etapa incluye igualmente obras de ingeniería civil. Así, la Emaap-Q ha instalado conexiones a lo largo de las quebradas para recoger las aguas usadas de las casas instaladas en las partes altas y evacuarlas hasta la red de saneamiento existente. El núcleo de este equipamiento parece haber sido la instalación de 7 kilómetros de colectores de evacuación de las aguas pluviales y usadas. Esta obra sirve para el buen funcionamiento de la red de saneamiento de la ciudad, pero no directamente para la lucha contra las amenazas. Al reducir la vulnerabilidad de esta red, se reduce también la vulnerabilidad del centro de Quito, pues la probabilidad

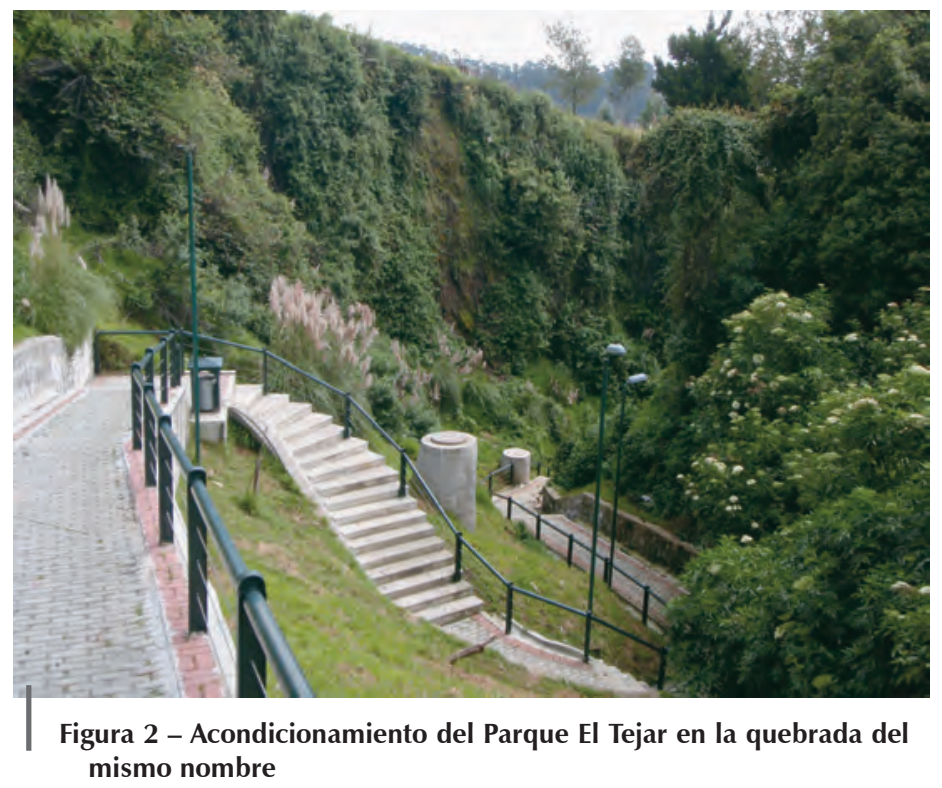

Las escalinatas que descienden hacia el centro histórico han sido acondicionadas en la quebrada cuyos taludes se han mantenido cubiertos de vegetación

Foto: A. Sierra, marzo de 2008 
de hundimientos y de desbordes de calzada es menor. En efecto, estos últimos provocan inundaciones cartografiadas en los documentos que acompañan el plan de reducción de los riesgos. Como la localización de las inundaciones se superpone en gran parte a la de algunos colectores, queda confirmado que el riesgo de una inundación depende estrechamente de la red de evacuación de las aguas, tanto en el centro histórico como en el norte de la ciudad. Aunque las autoridades difunden una representación del riesgo en el corazón de la ciudad, califican más claramente como en riesgo a las poblaciones instaladas hacia arriba.

Significativamente, esta fase del programa de lucha contra el riesgo se intitula «plan de mitigación y reasentamiento de familias en riesgo». En efecto, la Emaap-Q establece un vínculo estrecho entre reducción de la vulnerabilidad y desplazamiento de algunos residentes. En consecuencia, la municipalidad ha reubicado a 43 familias instaladas al borde de las quebradas profundas. A las otras casas se les ha reducido el terreno construible para dejar una franja de seguridad y para instalar allí rejas de protección y muros de contención (fig. 3). Estas rejas impiden caer a la quebrada y arrojar basura. Estas acciones han sido realizadas en concertación con los habitantes. Por último, 94 familias han recibido información y capacitación para prevenir accidentes.

Aquí están la mayoría de las quebradas abiertas. A diferencia de las laderas noroccidentales, las partes no rellenadas son menores y más encajonadas, pues

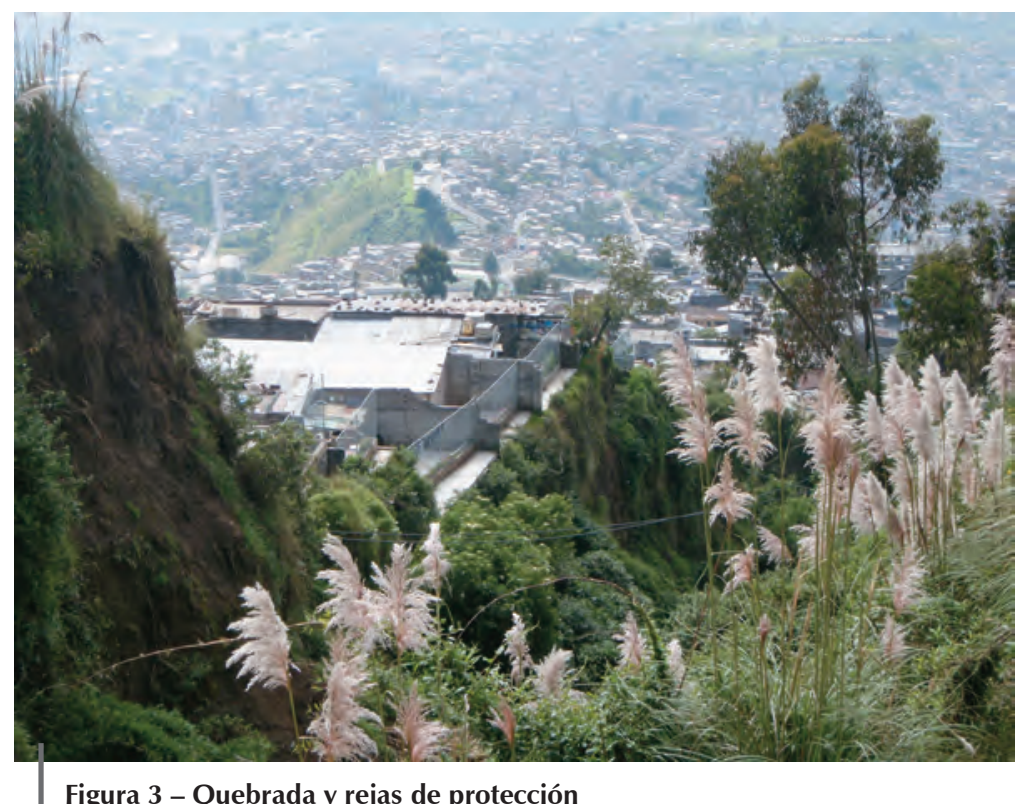

Figura 3 - Quebrada y rejas de protección

Las rejas han sido instaladas detrás de los límites de la parcela. El barrio está en plena urbanización. Un poco más arriba se encuentra un mirador con área de picnic.

Foto: A. Sierra, marzo de 2008 
las laderas centrales están más densamente urbanizadas. Esta condición local de desarrollo limita el acondicionamiento de obras de protección de gran tamaño, tal como han sido concebidas en el norte de la ciudad.

Es así como este acondicionamiento, menos pesado que en la primera fase del programa, está más integrado a una política de desarrollo urbano local y de mejoramiento del marco de vida. Este reduce parcialmente la exposición al peligro (expropiaciones, retiro de los bordes) y busca más claramente impedir nuevas instalaciones sobre las laderas más inclinadas y al borde de las quebradas. Esta política tiene como objetivo impedir la evacuación salvaje de las aguas usadas y la utilización de las quebradas como vertedero. Aunque esto no sea declarado públicamente, el conjunto de estas medidas demuestra la voluntad de reducir el daño de la red de evacuación de las aguas usadas y pluviales. La lógica inicial sigue siendo la misma, pero las modalidades de aplicación han cambiado, tanto a causa del contexto urbano como de las críticas que habían acompañado a la primera fase.

\section{3. El acondicionamiento de las laderas sudoccidentales (a partir de 2009)}

La extensión de la obra hacia las laderas sudoccidentales muestra, por un lado, el mantenimiento de la lógica de conjunto y, por otro, una nueva inflexión. La metodología adoptada prolonga aquella realizada en el centro, sobre todo por la idea de que es necesario integrar a la población en el tratamiento de los espacios. Esta es una ocasión para el PSA profundizar la reflexión sobre la vulnerabilidad de las poblaciones, aplicando un Índice de Vulnerabilidad Social (IVS). Este busca clasificar a las poblaciones que viven a proximidad de las quebradas según tres niveles de vulnerabilidad, del mayor al menor. A estas tres categorías corresponden tres niveles de ayuda que hay que aportar a la población. Así, la vulnerabilidad se convierte en un instrumento de asistencia pública: las poblaciones evaluadas como las más vulnerables reciben una ayuda financiera y técnica superior. Sin embargo, los criterios para calcular la vulnerabilidad social muestran que no es tanto la vulnerabilidad frente a los eventos morfoclimáticos la que entra en juego, sino la que se refiere a la integración social. Para tomar solo un ejemplo, las mujeres y las poblaciones afroecuatorianas reciben un peso superior en el cálculo del índice. Este valor discriminante (matemáticamente hablando) se justifica por el menor acceso de estas categorías sociales a la educación y al empleo. Estas también tienen ingresos más escasos que el promedio. Ahora bien, estos criterios están ya definidos: el censo de la población efectuado ad hoc distingue, por ejemplo, cuatro niveles de educación y cinco niveles de consumo, atribuyéndosele a cada uno niveles de vulnerabilidad en peso. Por ende, cada residente tiene un IVS que incluye estas variables. Así, los niveles de educación y de pobreza han sido retenidos dos veces en el caso de las mujeres y de los afroecuatorianos. En efecto, las autoridades dan a estos dos componentes una ponderación superior porque son considerados como más pobres y menos educados. Dicho de otra manera, una 
mujer rica y educada será considerada vulnerable porque las mujeres en general son consideradas como pobres y menos educadas. Por lo tanto, estas categorías de la población tienen un índice de vulnerabilidad superior: efectivamente es el caso en relación con la posición que estas ocupan dentro de la sociedad ecuatoriana. Sin embargo, ¿se puede afirmar que estas son más vulnerables que el promedio de la población frente a eventuales accidentes? Un ama de casa, por ejemplo, ¿no conoce mejor el medio ambiente del barrio que el hombre que trabaja lejos? O un hombre negro, zacaso es más vulnerable en caso de accidente que un miembro de otra comunidad? A pesar de la ambigüedad en su uso, estos ejemplos muestran que progresivamente se ha ido tomando en cuenta la noción de vulnerabilidad en el tratamiento de espacios geográficos para la lucha contra el riesgo urbano. La inflexión con respecto a la primera fase (el tratamiento de las laderas noroccidentales) está más clara. Sin embargo, son limitadas las operaciones de reubicación y, por ende, de reducción de la exposición al peligro de las poblaciones que provienen de ella: 14 familias deben abandonar los bordes de las quebradas. En cambio, el conjunto de las otras medidas dirigidas a la población atañe a un total de 205 familias. Estas recibirán ya sea una asistencia para reducir su vulnerabilidad ahí donde están instaladas o medidas de acompañamiento en su nuevo lugar de residencia. La operación consiste entonces en la difusión de información y en la realización de talleres de capacitación para la prevención y el manejo de crisis.

Si la dimensión social está bien presentada en el programa, no cabe ocultar la dimensión física del tratamiento contemplado en las laderas del sureste de la ciudad. En este nivel, igualmente hay que notar una inflexión de la política de lucha contra el riesgo. A diferencia de las dos primeras etapas, el relleno de las quebradas pasa a ser un componente central del programa. Esta medida es sorprendente porque numerosos estudios científicos locales han mostrado desde fines de los años 1970 que el relleno reduce el drenaje natural de la ciudad y provoca, en consecuencia, el desborde de las crecidas de lodo sobre las vías. Pese a ello, la práctica del relleno nunca ha sido interrumpida. Está enmarcada y depende de evaluaciones y de autorizaciones de la Emaap-Q. En el caso de las laderas sudoccidentales, parece que las quebradas que hay que rellenar no han sido consideradas peligrosas por carecer de un perfil muy activo (pocas crecidas, escasa erosión lineal y poco transporte de material). En numerosos casos, el relleno debía cubrir nuevos colectores. Más generalmente, este servirá para producir suelo urbano dentro de la lógica tradicional del desarrollo urbano quiteño: crear un continuum urbano y facilitar la movilidad. A la inversa de las laderas centrales, las quebradas son abiertas en su mayoría. El hecho de rellenarlas muestra la dinámica de urbanización de esta parte de la ciudad. La configuración permite construir ahí numerosas obras hidráulicas, entre las cuales se encuentran 13 pequeñas presas de embalse. Aunque han sido tomadas en cuenta las características sociales y una definición más amplia de la vulnerabilidad, esta etapa muestra que la lógica sigue siendo la de la lucha contra la amenaza natural. También se evidencia, sin embargo, que esto no se opone a las dinámicas históricas de construcción del riesgo (producción de suelo urbano mediante relleno). 


\section{LUCHA CONTRA EL RIESGO, INTEGRACIÓN DE LAS MÁRGENES URBANAS, REDUCCIÓN DE LA VULNERABILIDAD URBANA}

\section{1. Elección del espacio urbano a tratar y vulnerabilidades apuntadas}

En base al estudio de esta política pública de lucha contra el riesgo se pueden retener varias ideas.

La primera radica en la elección del espacio urbano a tratar. Considerando globalmente la ciudad, estamos en presencia de tres espacios de riesgo (fig. 4):

- las laderas, espacio de génesis de los eventos morfoclimáticos;

- el espacio urbano integrado a la planificación, que llamaremos por comodidad la ciudad baja: es un espacio de elementos esenciales, donde se concentran las actividades y los bienes afectados por los eventos morfoclimáticos y que se halla dentro de los límites oficiales de la ciudad;

- la margen urbana entre las laderas y la ciudad baja: es un espacio en parte ocupado ilegalmente y un frente de expansión urbana; está representado a la vez, como espacio de génesis de las amenazas según las autoridades y como espacio vulnerable, por el hecho de sufrir estas amenazas.

El límite entre estos tres espacios es confuso: la mayor parte de las laderas urbanizadas ya pertenece a los límites legales de la ciudad. El espacio urbano situado por encima del corredor occidental ha sido integrado progresivamente a la planificación urbana, perteneciendo además al espacio tratado por el programa de lucha contra el riesgo. El límite urbano oficial es un espacio de tensión porque es siempre considerado como un frente de urbanización con ocupaciones ilegales.

La política pública de lucha contra el riesgo quiso tratar los dos primeros espacioss, ambos considerados como espacios de génesis de las amenazas. Lógicamente, las autoridades municipales (y en primer lugar la Emaap-Q) han buscado reducir e incluso anular la fuerza de los fenómenos destructores, acondicionando estas laderas. A lo que se apunta es a la protección de los bienes de la ciudad baja; en particular a impedir que la movilidad y la red de saneamiento dejen de funcionar. El estudio de las representaciones espaciales del riesgo ha mostrado incluso que la portadora del riesgo es la margen intermedia entre lo urbano y lo «natural»: la población instalada sobre estas laderas es acusada de impermeabilizar y desestabilizar los suelos a través de la tala del bosque de eucalipto y de los terraceos que realiza de manera ilegal.

Por cierto, la población instalada sobre estas laderas parece más vulnerable que el resto de la población urbana, pues la pobreza, la dificultad de acceso, la precariedad y la ausencia de ciertos servicios son algunas de sus características. La

5 Únicamente la construcción de nuevos colectores en la parte central participa en el acondicionamiento de la ciudad baja. 


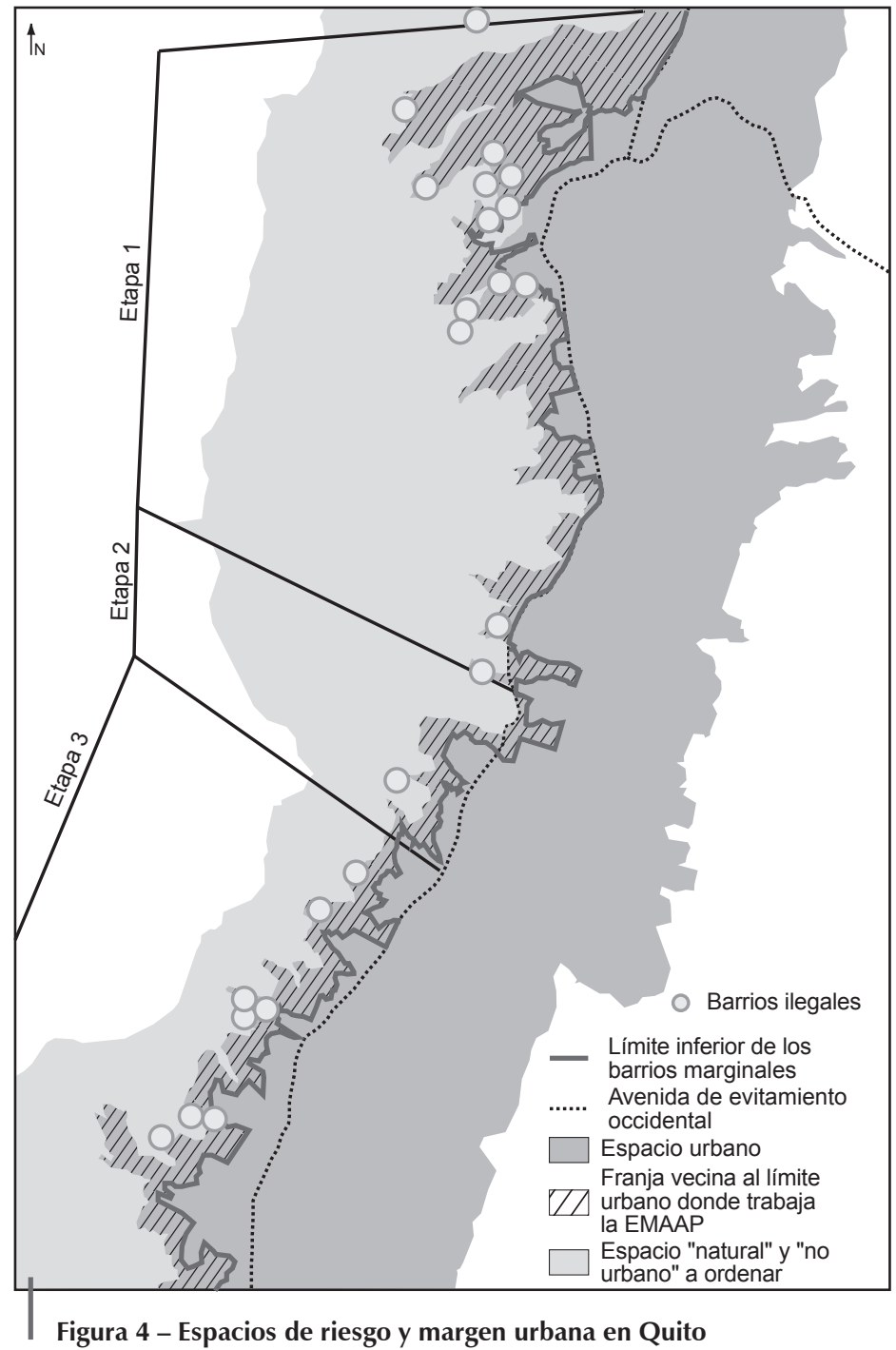

Fuente: A. Sierra a partir de documentos del PSA, 2009

política pública de tratamiento de estos espacios busca la reducción de algunos factores de vulnerabilidad a través de la disminución puntual de la puesta en exposición mediante las reubicaciones y de la difusión de la información sobre los riesgos y talleres de capacitación. Busca también mejorar las condiciones de vida que atañen particularmente a la higiene (gestión de los residuos, limpieza de las quebradas, conexión de recojo de las aguas usadas, etc.). Los últimos desarrollos de esta política muestran igualmente una integración de la idea de vulnerabilidad social en el tratamiento de estos espacios. A pesar de estos componentes del 
programa, el análisis multiescalar parece sin embargo indicar que no es tanto la vulnerabilidad de la población de las laderas la que es tratada, sino la de la ciudad que está al pie de las laderas. Esta conclusión se deduce más claramente de las obras efectuadas en el norte, donde el componente social es reducido, que de aquellas en el centro y en el sur, donde la población ha sido involucrada de manera más estrecha.

La protección de la red de saneamiento es uno de los hilos directores de esta política de reducción de riesgos. El rol de la Emaap-Q como directora de la política de lucha contra el riesgo morfoclimático sugiere esta orientación. Las acciones descritas confirman esta hipótesis. Aunque los acondicionamientos realizados reducen globalmente el riesgo, todos tienen como punto en común la reducción del daño a la red de saneamiento municipal: regularización de la cantidad de agua pluvial que hay que evacuar, limitación del material vertido en las quebradas y acarreado por las crecidas, limitación de la erosión lineal o de los derrubios de las quebradas, refuerzo de las estructuras de evacuación de las aguas pluviales o usadas, protección de las entradas de los colectores de agua pluvial, supresión de las evacuaciones salvajes de aguas usadas, etc. Reducir los derrames en las quebradas significa reducir los daños físicos y legales de la red de evacuación de las aguas. Reducir la presencia de las poblaciones sobre las laderas significa reducir estos derrames y los factores de erosión (según la representación que se tiene de las causas de las amenazas). También significa limitar la extensión de la red hacia las laderas y, por consiguiente, reducir los costos de inversión y los riesgos de ruptura de esta red. Esta opción de política pública se explica por el rol de la red manejada por la Emaap-Q en la construcción de la vulnerabilidad urbana. La red combinada de evacuación de las aguas ha sido colocada en las quebradas, contribuyendo así a su relleno. Su desarrollo, entonces, ha contribuido a hacer desaparecer el drenaje natural del sitio y a provocar las inundaciones de calzada. Esta red representa así un punto débil del sistema urbano, lo cual queda confirmado con el accidente del 30 de marzo de 2008 en el que se derrumbó una parte del intercambiador vial del Trébol.

Así, la Municipalidad ha hecho suya una política de lucha contra el riesgo que apunta prioritariamente a reducir la vulnerabilidad de una de sus redes. Esta elección fue integrada fácilmente porque siguió, a su vez, una lógica secular de tentativa de control de la urbanización hacia las laderas y de patrimonialización de sus espacios urbanos.

En efecto, ya sea sobre las laderas noroccidentales o centrales, las acciones efectuadas tratan de fijar un límite a la urbanización de las laderas. Si bien los casos de expulsión son limitados, todos los acondicionamientos tienden a impedir nuevas instalaciones. Las grandes obras de ingeniería, en particular las presas de embalse, fijan límites físicos y visibles a la urbanización. Por cierto, esta expansión urbana los rodea en las subcuencas más grandes como en la de la quebrada Rumihurcu. Sin embargo, las obras están acompañadas de hitos con los colores

6 Ver en este volumen el artículo de D. Salazar et al., pp. 561-572. 
de la ciudad, que indican el límite altitudinal de constructibilidad. Estos hitos se encuentran a lo largo de todas las laderas (fig. 5). Las autoridades han instalado igualmente paneles de señalización que alertan sobre los peligros que existen al instalarse más allá de este límite.

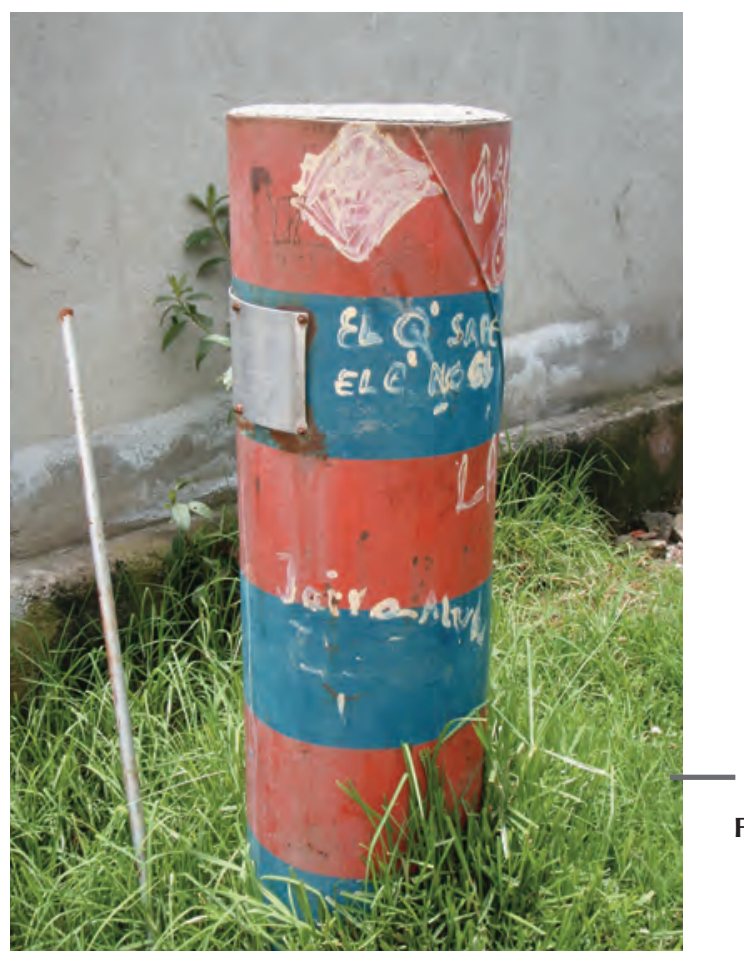

Figura 5 - Hito con los colores de la municipalidad que marca el límite legal de urbanización

Foto: Alexis Sierra, marzo de 2008

\section{2. Una planificación que ha integrado recientemente la dimensión riesgo}

La política actual de lucha contra el riesgo acompaña a una visión planificadora construida de manera duradera, en donde el riesgo no interviene como argumento sino en un periodo reciente que comenzó a fines de los años 1970. El primer plan de planificación, elaborado por el arquitecto Jones-Odriozola, preveía hacer inconstruibles las laderas, pero no hace ninguna mención de los riesgos de origen natural, y ni siquiera el volcán Pichincha es descrito como tal. Dominaron más las motivaciones estéticas e higienistas para no hacer de las laderas sino un vasto espacio de recreación, un telón de fondo de la ciudad acondicionado con senderos y lugares panorámicos. Los acondicionamientos actuales de las laderas centrales corresponden perfectamente a esta visión. La visión estetizante de las laderas sigue siendo de actualidad al estar hoy integrada a un proceso de patrimonialización más sistemático. El acondicionamiento de los parques sobre las laderas centrales 
prolonga la rehabilitación ejemplar del centro histórico, primer centro urbano clasificado como patrimonio mundial de la Unesco en 1978. Un poco más al norte, la inauguración en 2005 de un parque de diversiones y de un teleférico sobre las laderas hace eco al texto de Jones-Odriozola, que asignaba a estas laderas una función recreativa. También refleja hoy la idea de que estas «laderas y quebradas son el más importante patrimonio natural de Quito y tienen un valor paisajístico, escénico y ambiental alto» y aquella de que «el volcán Pichincha es un símbolo nacional, forma parte de la historia y de la identidad de la ciudad»». Todos estos elementos son confirmados por las múltiples representaciones iconográficas, particularmente pictóricas y turísticas, de las laderas.

Esta representación de las laderas ha adquirido una nueva dimensión desde los planes de planificación de 1967, 1971 y 1980, que han clasificado a su conjunto como cinturón verde. Estos «espacios verdes» representan entonces el pulmón de la ciudad en un contexto de muy fuerte expansión urbana. El proyecto de clasificación tal como es descrito en 1976 no menciona, sin embargo, el riesgo de origen morfoclimático. El acondicionamiento de un cinturón verde está justificado por razones de marco de vida (preservación del paisaje y aporte en oxígeno) y por razones de control de la expansión urbana. En efecto, los primeros suburbios (asentamientos marginales) se hicieron particularmente visibles sobre las laderas a comienzos de los años 1970. Tanto la prensa como los representantes elegidos denuncian regularmente la urbanización, por considerarla «anárquica»; es decir, esta aparece como incontrolable y fuera de cualquier planificación. El «cinturón verde» representa entonces un medio para dar una forma a la ciudad y para estar, al mismo tiempo, en sintonía con las preocupaciones medioambientales que emergen fuertemente en esa época. Hay que esperar el inicio de los años 1980 para que el riesgo de origen hídrico y morfoclimático se convierta en un argumento de acondicionamiento de las laderas. En 1983, el Estado dicta una ley que crea los «bosques protectores», herramienta de acondicionamiento destinada a reducir la erosión y los movimientos de masa. Dos accidentes ocurridos en Quito en 1983 precipitan la decisión de clasificar a las laderas occidentales como bosque protector. Los debates en el parlamento muestran que los argumentos ambientalistas (lucha contra el riesgo y preservación de los espacios verdes) se mezclan estrechamente con las consideraciones urbanísticas y sociales (control de la expansión urbana y en particular de la instalación ilegal de población pobre sobre las laderas). Cabe destacar la ambivalente representación de la parte no construida y protegida de las laderas: tanto como espacio de génesis de los peligros que amenazan a la ciudad, como espacio de protección de esta misma ciudad (justamente así lo sugiere la denominación de «bosque protector»). De esta manera, el código municipal del DMQ modificado en 20058, establece 6 categorías de áreas naturales. Entre estas, la categoría «vegetación protectora y

7 Introducción del informe del Programa de Saneamiento Ambiental («Mejoramiento y ampliación del control a toda el área natural», 2008).

8 Ordenanza Municipal 213 sustitutiva del Título V «Del Medio Ambiente», Libro Segundo del Código Municipal para el DMQ. 
manejo de laderas» designa un área que tiene por función el ser «una barrera de protección y de reducción de riesgos para la ciudad»»9. La constitución de un espacio verde a lo largo de las laderas busca a la vez valorizar el paisaje, limitar la urbanización y reducir la vulnerabilidad de la ciudad. La dificultad radica entonces en determinar un límite a este cinturón protector y en hacerlo respetar.

\section{3. Delimitación territorial de la ciudad y del riesgo}

A fines de los años 1970, el proyecto de corredor periférico vial fue presentado como un medio para fijar este límite y, en consecuencia, para dar una forma a la ciudad. En realidad este favoreció sobre todo la expansión urbana hacia las laderas, haciéndolas más accesibles y abriendo nuevos terrenos para la urbanización. Observemos, sin embargo, que frente a los eventos morfoclimáticos, este corredor tiene la ventaja de romper y de hacer divergir los flujos que descienden rápidamente de las pendientes, como lo muestra el ejemplo de la lava torrencial de La Comuna (Perrin et al., 2000). Este corredor facilita también el acceso de los auxilios. Indirectamente, este acondicionamiento urbano funciona a la vez sobre la vulnerabilidad y la resiliencia de la ciudad. Aunque el corredor no ha sido útil para materializar el límite de la ciudad, sí ha servido sin embargo como límite inferior al programa de acondicionamiento de las laderas en el marco de la lucha contra los riesgos de origen morfoclimático, tanto en el norte como en el sur.

A falta de poder ser materializado, el límite urbano es progresivamente definido por la reglamentación. En 1993, el Instituto Ecuatoriano Forestal y de Áreas Naturales y Vida Silvestre (Inefan) y la municipalidad de Quito fijan de manera «intangible» nuevos límites al «bosque protector», es decir a las áreas protegidas inconstruibles. Desde 2001, los diferentes planes no solo retoman estos límites sino que los extienden al sur para crear un espacio protegido continuo entre el macizo del Pichincha y el del Atacazo en el extremo suroeste de la ciudad10. Como esta definición jurídica del límite de constructibilidad era insuficiente, pues la dinámica ilegal de urbanización la traspasaba, las autoridades buscaron materializar sobre el terreno los límites de la ciudad. Ese es el rol de los hitos con los colores de la ciudad colocados en la primera etapa de la obra a fin de hacer visible el límite de constructibilidad. Su consecuencia ha sido estigmatizar como espacios de riesgo la porción del territorio municipal situada por encima de este límite (Sierra, 2009). Esta empresa de delimitación territorial del riesgo continúa actualmente, pues la Emaap-Q busca que este límite sea más visible. El objetivo sigue siendo impedir nuevas construcciones hacia arriba, dejando de lado uno de los argumentos de los nuevos ocupantes: el de desconocer el trazado de este límite. Así, existen estudios en curso para dejar una huella en el paisaje que permita separar claramente la ciudad de la no ciudad; la ciudad segura de la naturaleza patrimonializada.

9 Art. 384.14 de la Ordenanza Municipal 213.

10 Plan Quito Siglo XXI Equinoccio 21, Plan de Uso y Ocupación de Suelo, Plan de Gestión Integral de la Biodiversidad del Distrito Metropolitano de Quito. 


\section{4. ¿Controlar el riesgo mediante el control de las márgenes?}

Este esfuerzo de delimitación territorial se ha acompañado desde un inicio por una tentativa de control y de vigilancia de las laderas (ver cuadro 1). Se han creado comisarías, aunque con recursos humanos limitados.

Cuadro 1 - Cronología del control institucional de las laderas

\begin{tabular}{|c|l|l|}
\hline Año & \multicolumn{1}{|c|}{ Organización } & \multicolumn{1}{c|}{ Competencias } \\
\hline 1999 & $\begin{array}{l}\text { Creación de la Comisaría de } \\
\text { Laderas Norte }\end{array}$ & $\begin{array}{l}\text { Controlar el respeto del uso del suelo y } \\
\text { sancionar la violación de la planificación } \\
\text { establecida. Financiada por la Emaap-Q. }\end{array}$ \\
\hline 2005 & $\begin{array}{l}\text { Integración de la Comisaría } \\
\text { Norte en el seno de la } \\
\text { Comisaría Metropolitana de de } \\
\text { Ambiente }\end{array}$ & $\begin{array}{l}\text { Competencia sobre el conjunto de los } \\
\text { territorios considerados como no urbanos. } \\
\text { Control del uso del suelo mediante } \\
\text { inspecciones y participación en el plan de } \\
\text { gestión de las áreas naturales. }\end{array}$ \\
\hline 2009 & $\begin{array}{l}\text { Creación de la Comisaría de de } \\
\text { Laderas Sur del Pichincha }\end{array}$ & $\begin{array}{l}\text { Control del uso del suelo mediante } \\
\text { inspecciones y participación en el plan } \\
\text { de gestión de las áreas naturales. Sanción } \\
\text { de las violaciones. Apoyo de la policía } \\
\text { metropolitana y nacional. Tentativa } \\
\text { para integrar el conjunto de los actores } \\
\text { presentes en el trabajo de control. }\end{array}$ \\
\hline
\end{tabular}

Fuente: Emaap-PSA (2008)

La política pública de lucha contra el riesgo morfoclimático no se limita entonces a un acondicionamiento físico del territorio. Las diferentes herramientas implementadas apuntan a tratar la imagen, el marco jurídico y la situación social de una franja de territorio que actualmente actúa como una margen urbana. Todo parece concurrir para querer dominar la expansión urbana mediante el control y el tratamiento de esta margen, incluso hasta haciéndola desaparecer. En efecto, el ideal territorial (Gervais-Lambony, 2003) de las autoridades quiteñas parece pasar por la delimitación de un límite definitivo entre un espacio urbano seguro hacia abajo y, hacia arriba, un espacio «natural» cuyas dinámicas serían dominadas y que estaría totalmente integrado al patrimonio de la ciudad. La actual margen intermedia, vulnerable porque está ocupada por una población pobre y precaria, debe integrar la ciudad normada, ya sea mudándose o ya sea trasformándose. Por lo tanto, hoy en día la vulnerabilidad de la población parece más un estigma que revela la vulnerabilidad de la ciudad y que es necesario combatir. 


\section{CONCLUSIONES}

Existe un vínculo estrecho entre la lucha contra los riesgos de origen natural y una forma de control social o de implementación del Estado de derecho. Actualmente, la municipalidad de Quito implementa un plan integrado de gestión de riesgos. Esta nueva etapa de la política pública de prevención ha sido institucionalizada mediante una ordenanza'11. El objetivo es hacer coherentes las acciones efectuadas por las diferentes instituciones municipales y tener una visión menos segmentada del riesgo. Es así como los riesgos de origen natural deben ser tratados en relación estrecha con los riesgos sociales (violencia, delincuencia, etc.). Este desplazamiento muestra que el riesgo es más comprendido a partir de la vulnerabilidad de la población, sea cual fuere la amenaza, que a partir de esta última. Geográficamente, se revela una superposición creciente entre espacialización del riesgo y espacialización de la seguridad; entre espacio urbano de riesgo y espacio urbano de no derecho, marginal, al que hay que convertir en seguro e integrarlo.

La política de lucha contra el riesgo muestra así lógicas planificadoras que se han desarrollado a largo plazo. Esta atestigua también la integración (por etapas sucesivas) de los conceptos y de las problemáticas del análisis del riesgo. Estas se traducen en una renovada fe en el control de las dinámicas naturales (lucha contra la amenaza, continuación de los rellenos y del modo de desarrollo urbano). Su corolario es una inversión espectacular del punto de vista de la responsabilidad frente al riesgo: no hay fatalidad de la naturaleza; por lo contrario, la amenaza natural es percibida como provocada por las poblaciones instaladas sobre las laderas. Por esta razón, las amenazas de la naturaleza terminan mezclándose estrechamente con las amenazas sociales para calificar los espacios de riesgo.

\section{Referencias citadas}

EMAAP-Programa de Saneamiento Ambiental, 2008 - Plan definitivo de mitigación y reasentamiento de familias en riesgo, 30 pp.; Quito: informe, 2009.

METZGER, P. \& PELTRE, P., 1996 - Gestion de l'environnement urbain et risques «naturels». La problématique des quebradas à Quito (Équateur). Bulletin de l'Institut Français d'Études Andines, 25 (3): 531-552. Boletín temático «Les risques naturels et leur gestion en Équateur. Diversité des exemples, complémentarité des approches» ( $R$. D'Ercole, ed.); Lima: IFEA, Orstom, Cifeg.

PELTRE, P., 1989 - Quebradas y riesgos naturales en Quito, periodo 1900-1988. In: Riesgos naturales en Quito, lahares, aluviones y derrumbes del Pichincha y del Cotopaxi: 4591; Quito: Corporación Editora Nacional, Colegio de Geógrafos del Ecuador.

11 Ordenanza Municipal 265, enero de 2009. 
La política de mitigación de los riesgos en las laderas de Quito: ¿qué vulnerabilidad combatir?

PERRIN, J.-L., FOURCADE, B., POULENARD, J. \& RICHARD, D., 2000 - Quito face à un risque d'origine naturelle : les laves torrentielles. Revue de Géographie Alpine, n. ${ }^{\circ}$ 2, tomo 88: 43-57.

SIERRA, A., 2000 - Gestion et enjeux des espaces urbains à risque d'origine naturelle : les versants et les quebradas de Quito, Équateur, 326 pp.; París: université de Paris VIII. Tesis de doctorado.

SIERRA, A., 2003 - La division sociale du risque d'origine naturelle à Quito, les boues de Quito, Équateur. Annales de la Recherche Urbaine, n. ${ }^{\circ}$ 95: 47-53.

SIERRA, A., 2009 - Espaces à risque et marges : méthodes d'approche des vulnérabilités urbaines à Lima et Quito. Cybergeo: European Journal of Geography [En línea], Dossiers, Vulnérabilités urbaines au sud, document 456, puesto en línea el 14 de mayo de 2009. URL: http://www.cybergeo.eu/index22232.html 